\begin{tabular}{cc} 
Sharif University of Technology \\
Scientia Iranica \\
SCIENTIA & Transactions F: Nanotechnology \\
I RAN I CA & http://scientiairanica.sharif.edu \\
\hline
\end{tabular}

\title{
Green synthesis of Ag nanoparticles by methadone and their cytotoxicity against human breast cancer cells
}

\author{
A. Shafaei ${ }^{a}$, G.R. Khayati ${ }^{\mathrm{b}, *}$, and R. Hoshyar ${ }^{\mathrm{c}, \mathrm{d}}$ \\ a. Department of Nanotechnology, Mineral Industries Research Center (MIRC), Shahid Bahonar University of Kerman, \\ 7618868366 , Kerman, Iran. \\ b. Department of Materials Science and Engineering, Faculty of Engineering, Shahid Bahonar University of Kerman, Kerman, Iran. \\ c. Department of Microbiology and Molecular Genetics, Biomedical Physical Sciences, Michigan State University, East Lansing, MI, \\ USA, 48824. \\ d. Cellular and Molecular Research Center, Department of Biochemistry, Faculty of Medicine, Birjand University of Medical \\ Sciences, Birjand, Iran.
}

Received 18 March 2020; received in revised form 15 June 2021; accepted 19 July 2021

\section{KEYWORDS \\ Silver nanoparticles; Cytotoxicity activity; Density functional theory; Green synthesis; Methadone syrup.}

\begin{abstract}
Due to their fundamental applications in medicine, preparation of silver nanoparticles (AgNPs) has recently improved using green technique. In this study, methadone syrup (ME) was used for preparing AgNPs as a reducing and stabilizing agent with the objective of in vitro cytotoxicity effect against human breast cancer cells. The characteristics of the prepared particles were investigated using Transmission Electron Microscopy (TEM), Environmental Scanning Electron Microscopy (ESEM), Energy-Dispersive X-ray Spectroscopy (EDS), Dynamic Light Scattering (DLS), UVVisible (UV-Vis) spectroscopy, Fourier Transform Infrared (FT-IR) spectroscopy, and XRay Diffraction (XRD) studies. AgNPs (about $18 \mathrm{~nm}$ ) were synthesized in a spherical and uniform distribution. The mechanism of ME through the synthesis was proposed based on FT-IR analysis and Density Functional Theory (DFT). To investigate the cytotoxicity of the prepared AgNPs using ME, 3-(4,5-dimethylthiazol-2-yl)-2,5-diphenyltetrazolium bromide (MTT) assay was employed in the range of $0-100 \mu \mathrm{g} / \mathrm{mL}$. As a function of its dosage, the green synthetic AgNPs exhibited anti-proliferative activities against MDA-MB-468 cells with respect to ME. The results pointed to the feasibility of producing AgNPs in a simple, rapid, and green manner using $\mathrm{ME}$, which has an important function in inhibiting the growth of breast cancer cells.

(C) 2021 Sharif University of Technology. All rights reserved.
\end{abstract}

\section{Introduction}

In recent years, metal nanoparticles have received significant attention in different medical and pharmaceutical fields owing to their low volume-to-surface

*. Corresponding author. Fax: +98-341-2114053 E-mail addresses: eng.amineshafaei@gmail.com ( $A$. Shafaei); khayatireza@gmail.com, khayati@uk.ac.ir (G.R. Khayati); reyhaneh.houshyar@gmail.com (R. Hoshyar)

doi: $10.24200 /$ sci. 2021.55674 .4345 ratios as well as their unique physical, chemical, and biological properties. Different methods have been proposed to prepare nanoparticle [1-3]; however, the chemical and physical techniques used to synthesize the nanoparticles are often very expensive. They also release residual toxic in the environment and sometimes make carcinogenic reactions. Under these conditions, the possibility of using these nanoparticles in medical applications is severely restricted. In some cases, reaction by-products tend to agglomerate nanoparticles and deteriorate their properties [4,5]. Therefore, the need for a low-cost method without 
producing toxic substances and causing environmental issues is strongly recommended. Green synthesis is a good alternative to conventional physical and chemical preparation techniques and enjoys several advantages such as biocompatibility and low production cost. Algae, fungi, plants, and bacteria are extensively used for preparing nanoparticles through green synthesis [68]. Among metallic nanoparticles, silver nanoparticles (AgNPs) are one of the most important materials that are extensively used in different pharmaceutical and healthcare industries. These nanoparticles remain in the solution with remarkable chemical stability in shape and size. The main reason for the widespread usage of these nanoparticles is their inherent antimicrobial, antifungal, antioxidant, and anticancer potential [9-11].

Cancer is a fatal disease with a high mortality rate. Genetic and environmental factors are important factors in cancer development. However, new targets for the treatment can be provided with the help of genetic, molecular, and cellular basis knowledge. One of the main purposes of delivering anti-cancer drugs is to deliver the drug to the cancer tissue and use the minimum concentration of drugs to reduce the toxic effects of drug on the normal cells. Therefore, it is possible to take advantages of the new drug delivery method with the help of nanoparticles [12]. Recently, use of methadone syrup (ME) in patients suffering from cancer has been considered. According to the studies conducted on cancer, ME can kill the cells; however, there has not been any clinical study on increasing survival possibility in cellular patients so far. ME is a drug with analgesic properties similar to morphine, which is also regarded as an analgesic in adult patients suffering from cancer. In addition, it is used in cancer patients who do not respond to morphine or opioids. The main contributions of the current study are: (i) preparation of AgNPs using green reduction technique with ME; (ii) investigation of cytotoxic activities of the final products against the human breast cancer cells (MDA-MB-468), and (iii) application of Density Functional Theory (DFT) to study the mechanism of $\mathrm{Ag}^{+}$reduction using $\mathrm{ME}$.

\section{Experimental}

\subsection{Material}

In this study, silver nitrate (Merck Company, 99.99\% purity) was used as Ag precursor. To provide the reducing and stabilizer agent, ME (Iranian food and drug administration) was utilized. $\mathrm{HNO}_{3}$ and $\mathrm{NaOH}$ (Merck Company, 65\%, 98\% purity, respectively) were employed to adjust $\mathrm{pH}$. Double distilled water was used as solvent. Human breast cancer cells (MDAMB-468; IBRC C10095) and human normal cells (MCF10A, A-375) were purchased from Iranian Bio- logical Resource Center (IBRC, Tehran, Iran). Further, 3-(4,5-dimethylthiazol-2-yl)-2,5-diphenylt etrazolium bromide (MTT) was prepared by Sigma-Aldrich (St. Louis, MO). Gibco (Invitrogen, NY, USA) provided Fetal Bovine Serum (FBS), Trypsin, PenicillinStreptomycin, and RPMI 1640 solutions.

\subsection{Preparation of AgNPs by green technique}

For green synthesis of AgNPs, $1 \mathrm{mM}$ aqueous solution of $\mathrm{AgNO}_{3}$ was prepared and used as $\mathrm{Ag}$ precursor. In the next step, $10 \mathrm{~mL}$ of $\mathrm{ME}$ with the feed rate of $9 \mathrm{~mL} / \mathrm{min}$ was added to $90 \mathrm{~mL}$ of $1 \mathrm{mM} \mathrm{AgNO}_{3}$ solution at the constant $\mathrm{pH}$ of 7 , temperature of $40^{\circ} \mathrm{C}$ for $30 \mathrm{~min}$, and speed of $130 \mathrm{rpm}$. The colorless solution gradually turned light yellow after $30 \mathrm{~min}$, indicating the complete formation of AgNPs. To obtain AgNPs, the final solution was washed three times with distilled water and centrifuged for $20 \mathrm{~min}$ at $15000 \mathrm{rpm}$. This step made it possible to remove water-soluble biomolecules. The purified particles were then dried for $24 \mathrm{~h}$ at $60^{\circ} \mathrm{C}$ on vacuum petri dishes for further analysis [13].

\subsection{Characterization of AgNPs}

Since the color change of the reaction mixture is a criterion for the formation of AgNPs, UV-Visible spectroscopy (UV-Vis) (Scan Drop, Analytic Jena Co, Germany) [14] was used to investigate the reduction process. The surface plasmon vibration of the final products at a wavelength of 200-700 $\mathrm{nm}$ was also investigated. To determine the biomolecules, from AgNPs prepared, Fourier Transform Infrared spectroscopy (FT-IR) (BRUKER, model TENSOR 27, Germany) was used. Phase determination of the products was done using X-Ray Diffraction (XRD) (Philips, X'pertMPD system by $\mathrm{Cu}-\mathrm{K}_{\alpha}$ ) pattern. Further, scanning was carried out in the range of $2 \theta=30-70^{\circ} \mathrm{C}$ at a constant time of $2 \mathrm{sec}$ and a scanning rate of $0.02^{\circ} / \mathrm{min}$. Scherrer equation [15] and Warren's method were employed in conjunction for calculating the size of crystalline AgNPs after the removal of the peak broadening of X-ray due to the instrumental error [15].

Scanning electron microscope analysis was performed using an Environmental Scanning Electron Microscopy (ESEM) (QUANTA 200, USA). Moreover, the elemental analysis was done using Energy Dispersive X-ray Spectroscopy (EDS) (EDS Silicon Drift 2017, USA), and the ESEM image and EDS spectrum of the synthesized AgNPs were recorded. The morphological features of nanoparticles were confirmed by Transmission Electron Microscopy (TEM) (PHILPS EM-208S) using an accelerated voltage of $200 \mathrm{kV}$. In addition, Dynamic Light Scattering (DLS) (Malvern Zetasizer Nano range instrument) was utilized to determine the particle-sized distribution of the prepared sample. 


\subsection{Cytotoxicity assay}

To determine the anti-proliferative activity of AgNPs and ME, MTT assay was used with consideration of cell viability as a criterion. RPMI 1640 medium (Inoclon, Iran) was prepared by a combination of 100 units $/ \mathrm{mL}$ penicillin, 10\% FBS serum, and $100 \mathrm{mg} / \mathrm{mL}$ streptomycin, and it was used to culture the cells in an atmosphere containing $5 \% \quad \mathrm{CO}_{2}$ at a temperature of $37^{\circ} \mathrm{C}$. Consequently, different concentrations of $\mathrm{ME}$ and ME-AgNPs $(0-100 \mu \mathrm{g} / \mathrm{mL})$ were prepared $(0-48$ h) and exposed to the cells. The absorbance values were considered to determine the cell viability using Eq. (1). Then, IC50, i.e., the drug concentration that reduced the absorbance of the treated cells by $50 \%$, was compared with that of the untreated cells, which was used as a criterion for the comparison of ME-AgNPs and ME behaviors against the MDA-MB-468 cells after $48 \mathrm{~h}$. Each trial was repeated three times, and mean $\pm \mathrm{SD}$ was used to obtain the final results. In case the value of $p$ is lower than 0.05 , it is considered significant. Finally, based on Eq. (1), the effect of the samples is expressed as the percentage of cell viability [16]:

$$
\begin{aligned}
& \% \text { Cell viability }= \\
& \frac{\text { Absorbance at } 570 \mathrm{~nm} \text { of treated cell }}{\text { Absorbance at } 570 \mathrm{~nm} \text { of control cell }} * 100 .
\end{aligned}
$$

\subsection{Quantum chemical studies}

The optimization of the geometry of the ME species was performed using the CP2K quantum chemistry package [17]. The Gaussian augmented plane waves [18] with a 300 Ry cut-off were also used. Calculations were completed using Goedecker-Teter-Hutter pseudopotentials [19] for core electrons along with the MOLOPT basis set of double zeta valence plus polarization [20]. Perdew-Burke-Ernzerhof (PBE) exchangecorrelation functional [21] was adopted for each calculation, as implemented in the CP2K package. The convergence limit for the self-consistent field calculation was set to $10^{-6}$. A consistent and accurate ab initio parametrization of Density Functional Dispersion correction (DFT-D) for 94 elements $\mathrm{H}-\mathrm{Pu}$ was considered [22]. The optimization of the geometry was done using Broyden-Fletcher-Goldfarb-Shannon (BFGS) algorithm [23-26] until the maximum force on each atom was lower than $10 \mathrm{meV} / \AA$ with a maximum step size of 0.002 B. Partial Density Of States (PDOS) of all optimized geometries was obtained to define each atom type contribution at the energy levels.

The difference between the energies of Highest Occupied Molecular Orbital (HOMO) and Lowest Unoccupied Molecular Orbital (LUMO) is called HOMOLUMO gap or frontier orbital energy gap. LUMO and HOMO are often regarded as frontier orbitals. In organic semiconductors, the former is similar to the conduction band level, while the latter is similar to the valence band level. Frontier orbital energy gap is indicative of the interaction between the molecules that can be considered as a criterion for the chemical reactivity of the molecules. In this study, the quantum chemical parameters such as Dipole moment (D), energy gap $(\Delta E)$, Energy of LUMO (ELUMO), and Energy of HOMO (EHOMO) were calculated using VESTA software (Ver. 3) [27].

\section{Results and discussion}

\subsection{UV-Vis Spectra}

First, AgNPs were synthesized by adding $9 \mathrm{~mL}$ ME to $90 \mathrm{~mL}$ of $1 \mathrm{mM} \mathrm{AgNO}_{3}$ solution at a stirring rate of $130 \mathrm{rpm}, \mathrm{pH}=7$, and temperature of $40^{\circ} \mathrm{C}$. The first sign of the preparation of $\mathrm{AgNPs}_{\mathrm{s}}$ is the discoloration of the solution. The bright yellow color obtained after 30 min confirmed the reduction of $\mathrm{AgNPs}_{\mathrm{s}}$ in the solution. The results of UV-Vis spectroscopy of nanoparticles synthesized by $\mathrm{ME}$ are illustrated in Figure 1. The maximum absorption peak $(415 \mathrm{~nm})$ in the curve obtained from the extract after the synthesis of AgNPs in the range of 400 to $450 \mathrm{~nm}$ confirmed the formation of $\mathrm{AgNPs}$ [28].

\subsection{Determination of functional groups in bio-reduction of AgNPs based on FT-IR spectrum}

To identify the functional groups responsible for limiting and stabilizing the AgNPs, infrared spectroscopy analysis was carried out in the range between 400 and $4000 \mathrm{~cm}^{-1}$. The FT-IR spectra of ME and green synthesized AgNPs are shown in Figure 2. As shown in the FT-IR spectrum of ME, there are strong absorption bands at 2931, 2369, 1636, 1351, and $1062 \mathrm{~cm}^{-1}$. The spectra of ME and synthesized AgNPs show a shift in the peaks. The peak at $2931 \mathrm{~cm}^{-1}$ is probably driven by asymmetric and symmetric stretching vibrations of the $\mathrm{C}-\mathrm{H}$ groups such as $\mathrm{CH}_{2}$ and $\mathrm{CH}_{3}$. The presence

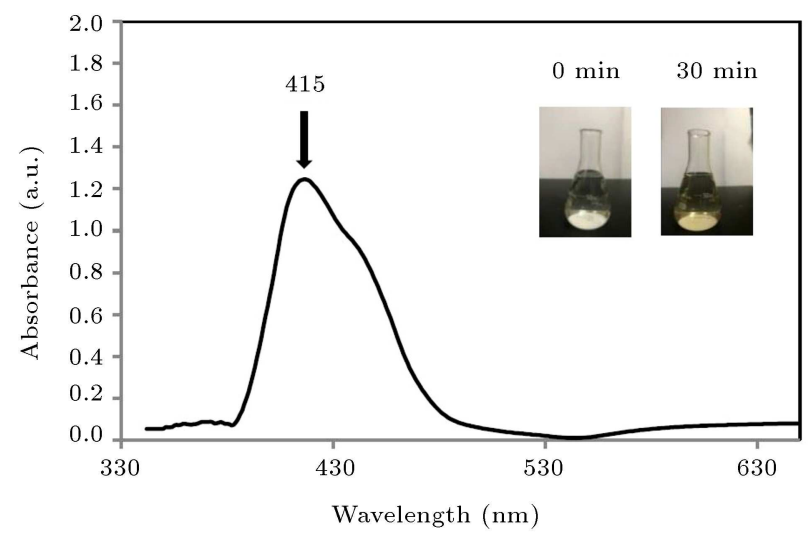

Figure 1. UV-Visible (UV-Vis) spectra of the synthesized AgNPs. 


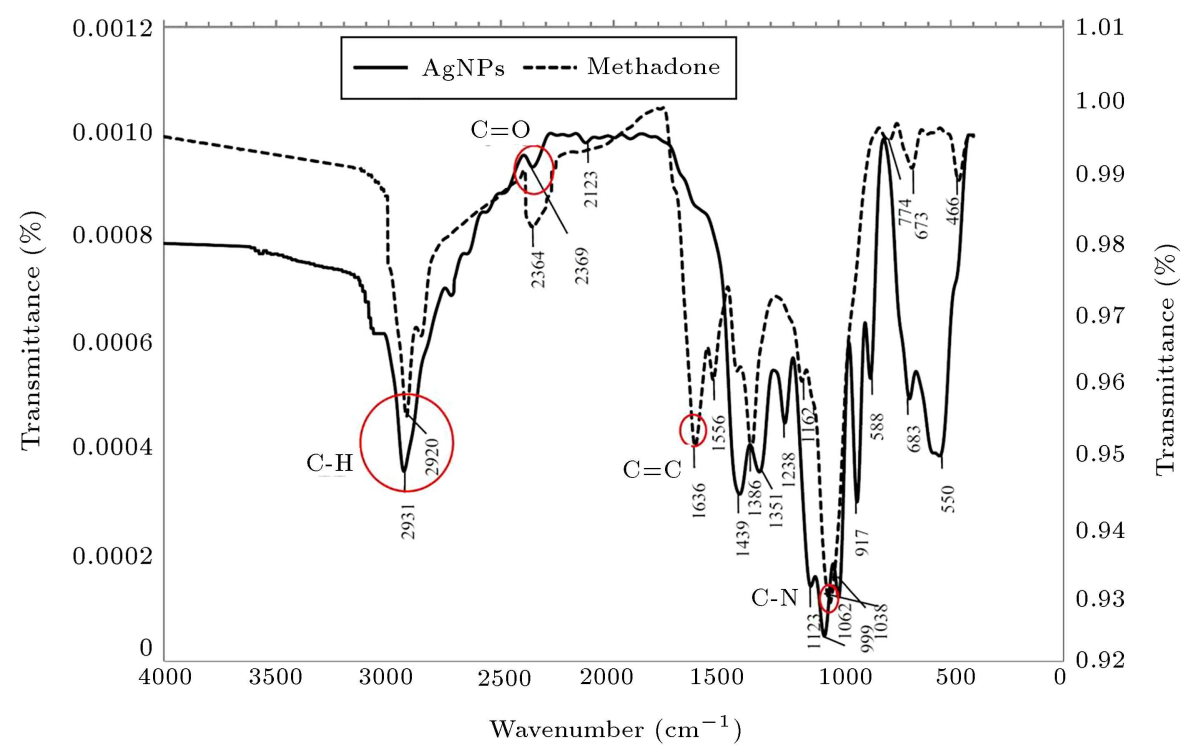

Figure 2. Fourier Transform Infrared (FT-IR) spectrum for ME and AgNPs; ME: methadone syrup.

of a peak at $2369 \mathrm{~cm}^{-1}$ in the AgNPs spectrum is indicative of the binding of some $\mathrm{C}=\mathrm{O}$ groups at the shell of the AgNPs resulting from the inherent characteristics of green synthesis with ME [29]. According to the literature [30], the peak at around 1636 $\mathrm{cm}^{-1}$ corresponds to the groups $\mathrm{C}=\mathrm{C}$ (around 1635 $\mathrm{cm}^{-1}$ ). The presence of the $\mathrm{NO}^{-3}$ ion is confirmed by the band located at $1351 \mathrm{~cm}^{-1}$ [31]. On the other hand, this peak is attributed to $\mathrm{C}-\mathrm{N}$ stretching (aromatic tertiary amines) $[32,33]$. The strong peak at $1062 \mathrm{~cm}^{-1}$ results from the $\mathrm{C}-\mathrm{N}$ stretching vibration of the amine group [34]. According to these data, the synthesized AgNPs contain the functional groups of tertiary amines, phenyl group, and ketone; therefore, immediately after the reduction of silver ions to $\mathrm{Ag}$, these groups are absorbed into the nanoparticles, thus making them stable. Hence, the ME can effectively reduce silver ions to AgNPs.

\subsection{ESEM, TEM, EDS, and DLS studies}

The ESEM images of AgNPs confirm the spherical morphology of the prepared particles (Figure 3(a)) with the average particle size lower than $20 \mathrm{~nm}$. In addition, EDS point chemical analysis (Figure 3(a)) confirms the formation of Ag. Moreover, EDS mapping of the prepared sample shows the homogenous distribution of AgNPs (Figure 3(c)). This can be concluded from the weaker signals of nitrogen, carbon, and oxygen with respect to Ag, especially at $3 \mathrm{keV}$ [35]. Such observations revealed that the synthesized AgNPs fitted successfully inside the nanoscopic ME templates through the green method. The images obtained from TEM of AgNPs also show that these nanoparticles have a relatively homogeneous spherical morphology with the mean size of about lower than $20 \mathrm{~nm}$ (Figure 3(b)). A closer look at the images of the TEM reveals that the edges of the nanoparticles are brighter than their core (Figure 3(b)). Based on Figure 3(c), it can be concluded that the edges of nanoparticles are surrounded by a layer of organic materials such as nitrogen, carbon, and oxygen. This layer acts as a stabilizing agent on the surface of green synthesized particles. Therefore, in addition to its reducing role, ME prevents the accumulation of particles (Figure $3(\mathrm{~b})$ ). The particle size distribution is given in Figure 3(e). The results from the DLS analysis indicate that the average size of the prepared particles is approximately $10 \mathrm{~nm}$.

\subsection{XRD studies}

The XRD pattern of AgNPs is depicted in Figure 4. The peaks at $2 \theta=32.29^{\circ}, 38.27^{\circ}, 44.48^{\circ}$, and $64.53^{\circ}$ correspond to the crystalline plates (111), (200), and (220) of the Ag phase with FCC structure, respectively. It should be noted that the diffraction peak at $32.29^{\circ}$ results from the crystalline and amorphous organic phase [36]. The average particle size of AgNPs was estimated to be about $18 \mathrm{~nm}$ with the consideration of the average value of (111), (200), and (220) peaks in Scherrer equation [37].

\subsection{Short term in vitro cytotoxicity assay}

The effects of the synthesized nanoparticles on the breast cancer were evaluated after $48 \mathrm{~h}$ at different concentrations using MTT assay and cell viability. The analysis of cytotoxic results showed that after $48 \mathrm{~h}$ incubation, ME and ME-AgNPs significantly prevented the growth of the MDA-MB-468 cell, i.e., cancer cells, as a function of ME/AgNPs dosage (Figure 5(a)). Also, the percentage of $\mathrm{ME}$ viability with $\mathrm{AgNPs}$ treated cells was lower than that of the ME treated cells at the same dosages. As shown in Figure 5(b), at low concentrations, the prepared nanoparticles (IC50=26.9 


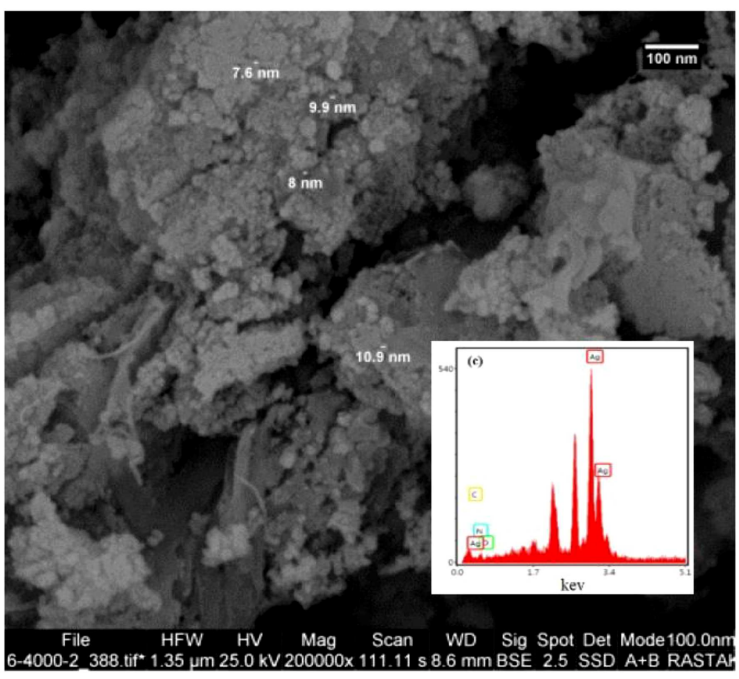

(a)

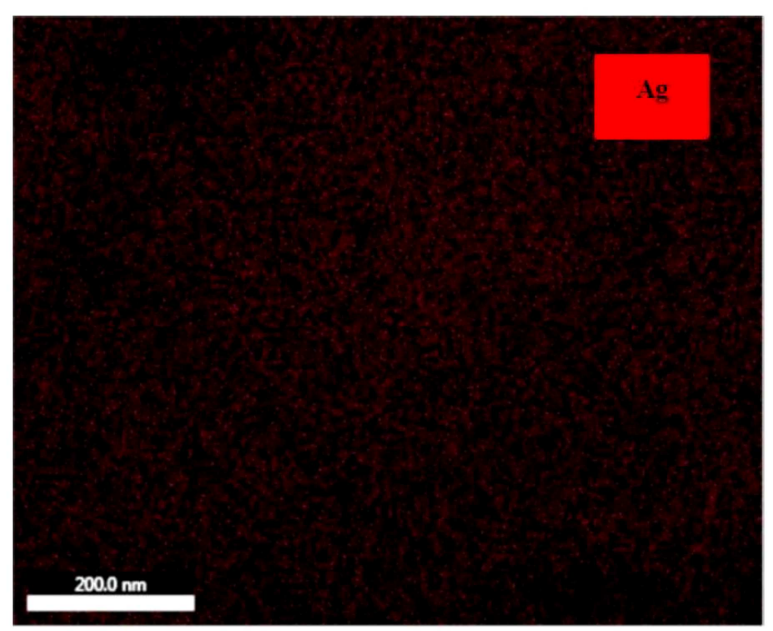

(c)

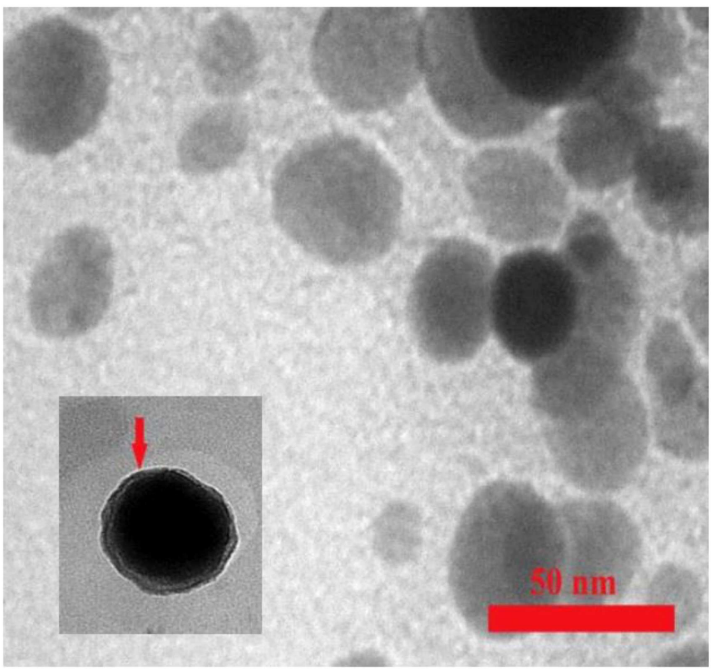

(b)

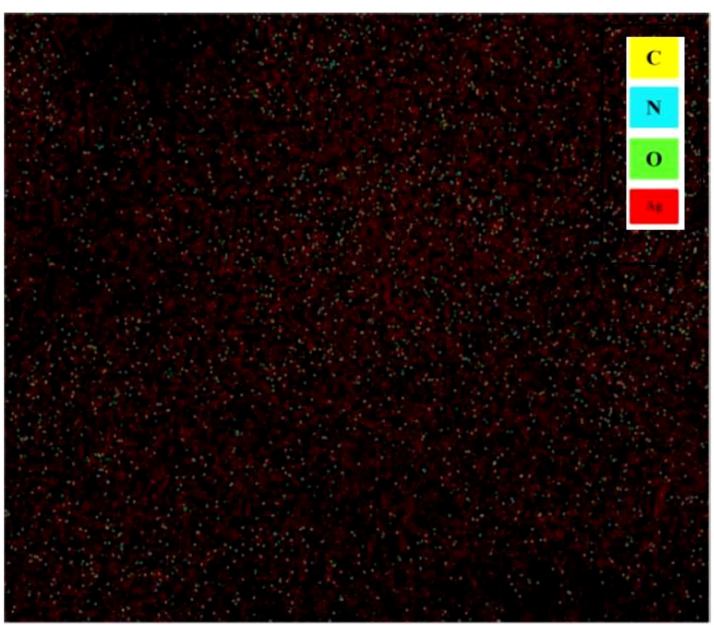

(d)

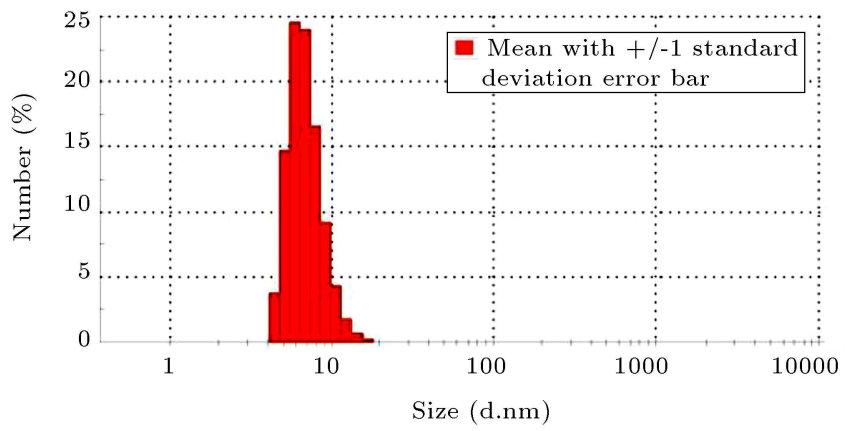

(e)

Figure 3. (a) Environmental Scanning Electron Microscopy (ESEM), (b) Transmission Electron Microscopy (TEM) image, (c, d) Energy-Dispersive x-ray Spectroscopy (EDS) mapping, and (e) Dynamic Light Scattering (DLS) of the synthesis of AgNPs using ME; ME: methadone syrup.

$\mu \mathrm{g} / \mathrm{mL}$ ) kill about $50 \%$ of cancer cells, which is 2.5 times less than the effect of $\mathrm{ME}$ at the same concentration. However, these treatments do not change the proliferation of MCF10-A cells, i.e., normal cells (Figure 5(c)).

\subsection{Quantum chemical study for the reduction of $\boldsymbol{A g N P s}$}

The optimized structures of the materials along with their HOMO and LUMO isosurfaces are depicted in Figure 6(a). The reaction mechanism can be explained 


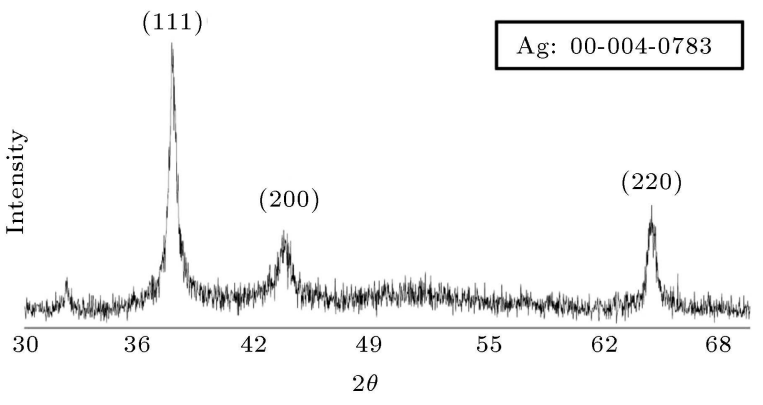

Figure 4. X-Ray Diffraction (XRD) pattern of the synthesis of AgNPs using ME; ME: methadone syrup.

through molecular orbital theory. Two molecules react when the electronic repulsion caused by charge attraction or orbital overlap is overcome by the amount of activation energy [36,38]. This energy is defined by the minimum difference between the energies of HOMO of a molecule and LUMO of the other. The orbital overlap is a necessary condition for a reaction to occur [38], and the closer the HOMO-LUMO energy of the two molecules is, the faster a reaction can start. In a green synthesis of AgNPs using ME, the minimum energy difference of the HOMO-LUMO of the $\mathrm{AgNO}_{3}$ and other compounds of $\mathrm{ME}$ are defined, and their energy levels are shown in Figure 6(b). The diagram shows that the HOMO of ME molecule is closer to the LUMO of $\mathrm{AgNO}_{3}$ in terms of energy (Figure 6(a)).

The amount of this energy is about $0.26 \mathrm{eV}$, indicating that the required energy to start the $\mathrm{Ag}$ reduction is small (Figure $6(\mathrm{~b})$ ). The minimum amount of energy needed for starting the Ag reduction through a reaction of $\mathrm{AgNO}_{3}$ and $\mathrm{ME}$ molecule is equivalent to the energy of an electromagnetic wave with the wavelength of about $4768 \mathrm{~nm}$ that lies in the infrared realm, indicating that the reduction can be performed even in case of sunlight with higher energy than the infrared waves. To determine which atoms contribute to the $\mathrm{AgNO}_{3}$ reaction with $\mathrm{ME}$, one should obtain the PDOS of the HOMO and LUMO energy levels, as shown in Table 1. As discussed earlier, the interacting orbitals of the reaction between $\mathrm{AgNO}_{3}$ and $\mathrm{ME}$ molecules were the HOMO of ME and LUMO of $\mathrm{AgNO}_{3}$.

As shown in Table 1, the LUMO of $\mathrm{AgNO}_{3}$ is mainly composed of an oxygen atom with a contribution of about $87 \%$, and the main contributor of the HOMO of $\mathrm{ME}$ is $\mathrm{N}$ and $\mathrm{C}$ atoms with about $57 \%$ and $27 \%$, respectively. It should be emphasized that $\mathrm{ME}$ is responsible for providing electrons, and the acceptable form is the $\mathrm{Ag}^{+}$ions in aqueous silver nitrate solutions. Based on the interactions between the donor and acceptor, the occurrence of the reduction of $\mathrm{Ag}^{+}$ions into $\mathrm{Ag}^{0}$ can be proven. This indicates that the $\mathrm{AgNO}_{3}$ molecule has an approximate ionic character and configures an ionic bond between the $\mathrm{Ag}$ atom, and the two other oxygen atoms are shown in

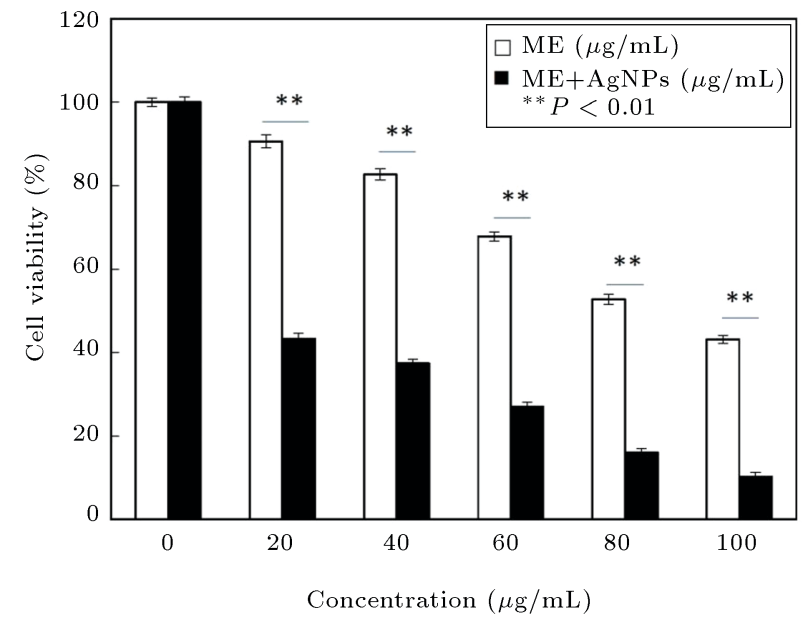

(a)

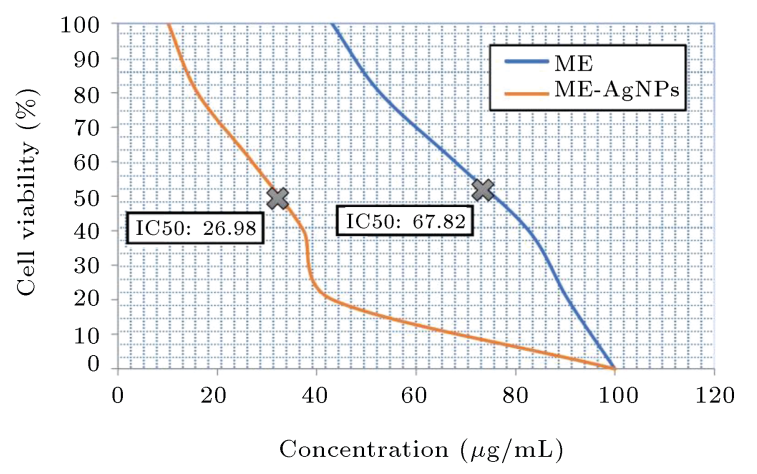

(b)

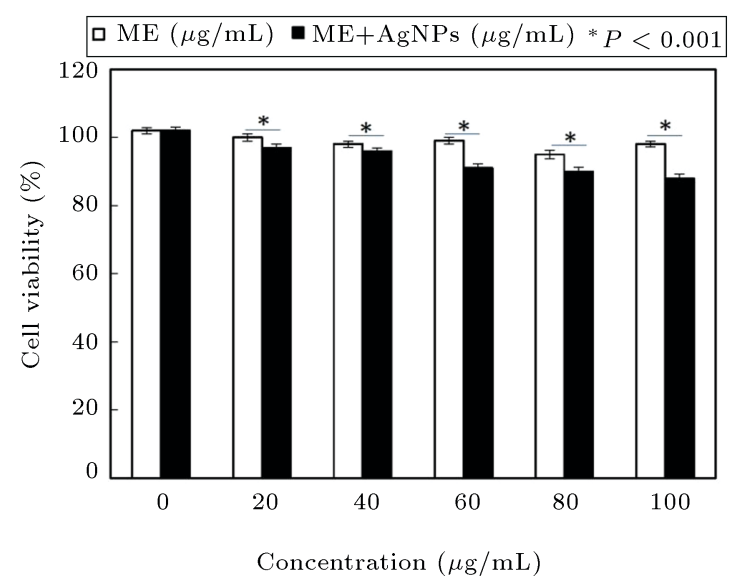

(c)

Figure 5. (a) MTT assay showing the cytotoxic effect of $\mathrm{ME} /(\mathrm{ME}-\mathrm{AgNPs})$ on MDA-MB-468 with ${ }^{* *} P<0.01$, (b) IC50 value of AgNPs against human breast cancer cells (MDA-MB-468), (c) MTT assay showing the cytotoxic effect of ME/(ME-AgNPs) on human normal cells (MCF10A) with ${ }^{*} P<0.001 ;$ ME: methadone syrup.

Figure 6(a). Therefore, $\mathrm{AgNO}_{3}$ molecules are dissolved in a polar solvent like water; hence, there is a possibility that $\mathrm{Ag}$ and $\mathrm{NO}_{3}$ components can be separated into $\mathrm{Ag}^{+}$and $\left[\mathrm{NO}_{3}\right]^{-}$. Indeed, ME HOMO gets closer to $\mathrm{AgNO}_{3} \mathrm{LUMO}$ and the oxygen atoms in $\mathrm{AgNO}_{3}$ form a 


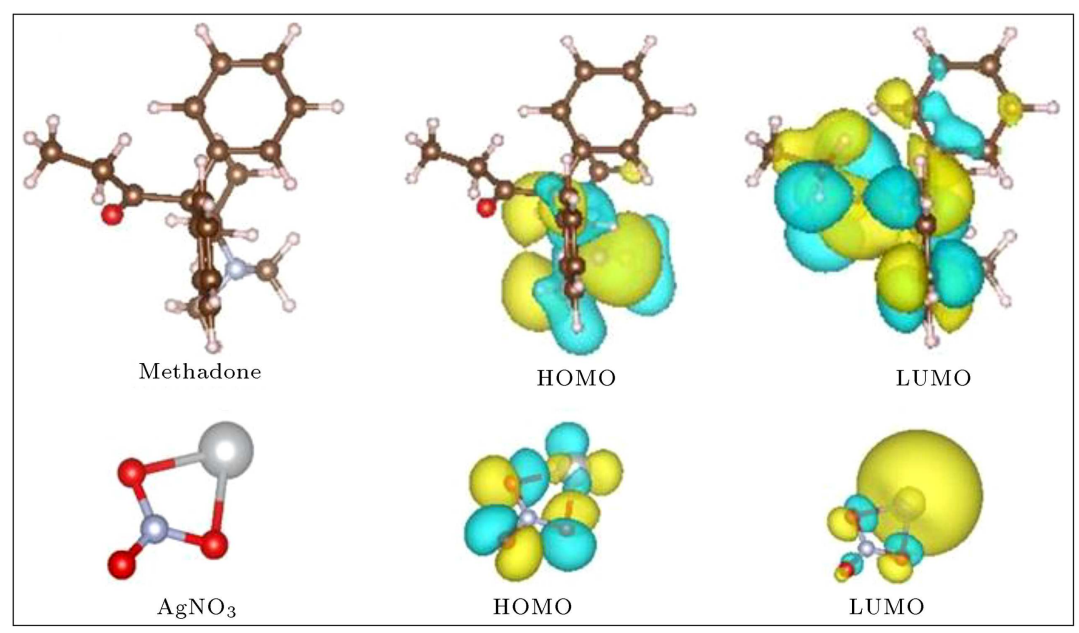

(a)

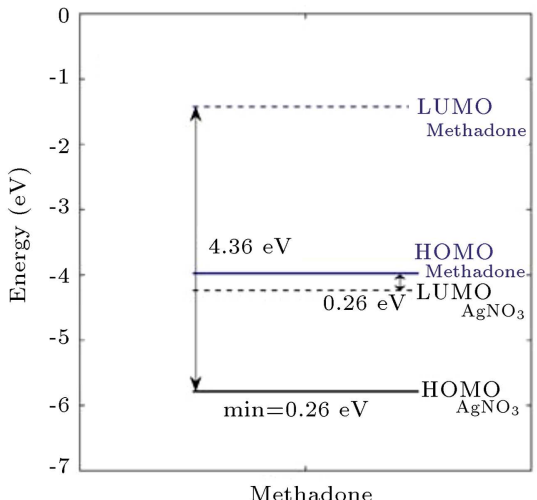

(b)

Figure 6. (a) The optimized structure, Highest Occupied Molecular Orbital (HOMO), Lowest Unoccupied Molecular Orbital (LUMO), and energy gap of the ME. (b) The HOMO and LUMO energy levels of $\mathrm{AgNO}_{3}$ (black lines) and ME (blue lines); ME: methadone syrup.

Table 1. Partial Density Of States (PDOS) of each molecule for their Highest Occupied Molecular Orbital (HOMO) and Lowest Unoccupied Molecular Orbital (LUMO).

\begin{tabular}{ccccccc}
\hline Molecule & Energy Level & Ag (\%) & O (\%) & N (\%) & C (\%) & H (\%) \\
\hline \multirow{2}{*}{ Methadone (ME) } & HOMO & - & 0 & 57.14 & 26.9 & 15.89 \\
& LUMO & - & 26.31 & 0 & 67.7 & 5.99 \\
\multirow{2}{*}{ AgNO $_{3}$} & HOMO & 84.40 & 15.43 & 0.17 & - & - \\
& LUMO & 1.49 & 86.84 & 11.67 & - & - \\
\hline
\end{tabular}

bond with the $\mathrm{C}$ and $\mathrm{N}$ atoms in the solution, meaning that the $\mathrm{NO}_{3}$ part of $\mathrm{AgNO}_{3}$ is absorbed by ME and $\mathrm{Ag}$ is departed. Of note, the presence of the NO3- ion is confirmed by the band located at $1351 \mathrm{~cm}^{-1}$ [31].

\section{Conclusion}

This study discussed the green synthesis of AgNPs by $\mathrm{ME}$ as a reducing agent. Different techniques including Transmission Electron Microscopy (TEM), Environmental Scanning Electron Microscopy (ESEM), Energy-Dispersive x-ray Spectroscopy (EDS), Dynamic Light Scattering (DLS), UV-Visible (UV-Vis), Fourier Transform Infraded (FT-IR), and X-Ray Diffraction (XRD) were used for characterization of the prepared AgNPs. The results illustrated that ME could successfully reduce $\mathrm{Ag}^{+}$into $\mathrm{Ag}$ with an average size of 18 $\mathrm{nm}$. The investigation based on Density Functional Theory (DFT) revealed that the oxygen atoms in $\mathrm{AgNO}_{3}$ formed a bond with $\mathrm{C}$ and $\mathrm{N}$ atoms in the solution; in other words, $\mathrm{NO}_{3}$ part of $\mathrm{AgNO}_{3}$ absorbed by $\mathrm{ME}$ and $\mathrm{Ag}^{+}$was reduced. The results from the MTT cell viability assay revealed that the prepared AgNPs using ME could effectively prevent the growth of human breast cancer cells as a dose-dependent manner. This study is limited in scope because of its disregard for animal models or human population because of addictive aspect or neurological effects of this component. In summary, it is possible to synergize the effect of $\mathrm{ME}$ as a painkiller and anti-proliferation agent in the presence of AgNPs through the green synthesis method.

\section{References}

1. Khandel, P., Yadaw, R.K., Soni, D.K., et al. "Biogenesis of metal nanoparticles and their pharmacological applications: present status and application prospects", J. Nanostructure Chem., 8(3), pp. 217-254 (2018).

2. Yaqoob, A.A., Ahmad, H., Parveen, T., et al. "Recent advances in metal decorated nanomaterials and their various biological applications: a review", Front. Chem., 8, p. 341 (2020).

3. Mousavi, S.M., Hashemi, S.A., Ghasemi, Y., et al. "Green synthesis of silver nanoparticles toward bio and medical applications: review study", Artif. Cells Nanomed. Biotechnol., 46(sup3), pp. S855-S872 (2018). 
4. Yan-yu, R., Hui, Y., Tao, W., et al. "Bio-synthesis of silver nanoparticles with antibacterial activity", Mater. Chem. Phys., 235, p. 121746 (2019).

5. Venugopal, K., Rather, H., Rajagopal, K., et al. "Synthesis of silver nanoparticles (Ag NPs) for anticancer activities (MCF 7 breast and A549 lung cell lines) of the crude extract of Syzygium aromaticum", $J$. Photochem. Photobiol., 167, pp. 282-289 (2017).

6. Erdogan, O., Abbak, M., Demirbolat, G.M., et al. "Green synthesis of silver nanoparticles via Cynara scolymus leaf extracts: The characterization, anticancer potential with photodynamic therapy in MCF7 cells", PloS One., 14, p. e0216496 (2019).

7. SP, V., Udayabhanu, U., and HS, L. "Plant-mediated green synthesis of Ag nanoparticles using Rauvolfia tetraphylla (L.) flower extracts: Characterization, biological activities and screening of their catalytic activity in formylation reaction", Scientia Iranica, 27(6), pp. 3353-3466 (2019).

8. Sattari, R. and Khayati, G.R. "Prediction of the size of silver nanoparticles prepared via green synthesis: A gene expression programming approach" , Scientia Iranica., 27(6), pp. 3399-3411 (2020).

9. Pawliszak, P., Malina, D., and Sobczak-Kupiec, A. "Rhodiola rosea extract mediated green synthesis of silver nanoparticles supported by Nanosilica carrier", Mater. Chem. Phys., 234, pp. 390-402 (2019).

10. Velgosova, O., Mrazikova, A., Cizmarova, E., et al. "Green synthesis of Ag nanoparticles: Effect of algae life cycle on Ag nanoparticle production and long-term stability", Trans. Nonferrous Met. Soc. China., 28, pp. 974-979 (2018).

11. Burduşel, A.C., Gherasim, O., Grumezescu, A.M., et al. "Biomedical applications of silver nanoparticles: An up-to-date overview", Nanomater., 8(9), p. 681 (2018).

12. Buttacavoli, M., Albanese, N.N., Di Cara, G., et al. "Anticancer activity of biogenerated silver nanoparticles: an integrated proteomic investigation", Oncotarget, 9, p. 9685 (2018).

13. Sufyani, A., Moslah, N., Hussien, N.A., et al. "Characterization and anticancer potential of silver nanoparticles biosynthesized from olea chrysophylla and lavandula dentata leaf extracts on HCT116 colon cancer cells", J. Nanomater, 2019, pp. 1-9 (2019).

14. Chokkalingam, M., Singh, P., Huo, Y., et al. "Facile synthesis of $\mathrm{Au}$ and $\mathrm{Ag}$ nanoparticles using fruit extract of Lycium Chinese and their anticancer activity", J. Drug Delivery Sci. Technol., 49, pp. 308-315 (2019).

15. Khayati, G.R. and Janghorban, K. "The nanostructure evolution of $\mathrm{Ag}$ powder synthesized by high energy ball millin", $J$. Drug Delivery Sci. Technol., 23, pp. 393-397 (2012). DOI: https://doi.org/10.1016/j.apt.2011.05.005

16. Mostafavinia, S.E., Khorashadizadeh, M., and Hoshyar, R. "Antiproliferative and proapoptotic effects of crocin combined with hyperthermia on human breast cancer cells", DNA Cell Bio., 35, pp. 340-347 (2016).
17. Hutter, J., Iannuzzi, M., Schiffmann, F., et al. "cp2k: atomistic simulations of condensed matter systems", Wiley Interdiscip. Rev.: Comput. Mol. Sci., 4, pp. 1525 (2014).

18. Lippert, B.G., Parrinello, J.H., and Michele, A. "Hybrid Gaussian and plane wave density functional scheme", Molecular Physics., 92, pp. 477-488 (1997).

19. Hartwigsen, C., Goedecker, S., and Hutter, J. "Relativistic separable dual-space Gaussian pseudopotentials from H to Rn", Physical Review B., 58, p. 3641 (1998).

20. VandeVondele, J. and Hutter, J. "Gaussian basis sets for accurate calculations on molecular systems in gas and condensed phases", J. Chem Phys., 127, p. 114105 (2007).

21. Perdew, J.P., Burke, K., and Ernzerhof, M. "Generalized gradient approximation made simple", Phys. Rev. Lett., 77, p. 3865 (1996).

22. Grimme, S., Antony, J., Ehrlich, S., et al. "A consistent and accurate ab initio parametrization of density functional dispersion correction (DFT-D) for the 94 elements H-Pu", J. Chem Phys., 132, p. 154104 (2010).

23. Broyden, C.G. "The convergence of a class of doublerank minimization algorithms 1 . general considerations", IMA J. Appl Math., 6, pp. 76-90 (1970).

24. Fletcher, R. "A new approach to variable metric algorithms", Comput J., 13, pp. 317-322 (1970).

25. Goldfarb, D. "A family of variable-metric methods derived by variational means", Math Comput., 24, pp. 23-26 (1970).

26. Shanno, D.F. "Conditioning of quasi-Newton methods for function minimization", Math Comput., 24, pp. 647-656 (1970).

27. Momma, K. and Izumi, F. "VESTA 3 for threedimensional visualization of crystal, volumetric and morphology dat", J. Appl Crystallogr., 44, pp. 12721276 (2011).

28. David, L. and Moldovan, B. "Green synthesis of biogenic silver nanoparticles for efficient catalytic removal of harmful organic dyes", Nanomater., 10(2), p. 202 (2020).

29. He, Y., Wei, F., Ma, Z., et al. "Green synthesis of silver nanoparticles using seed extract of Alpinia katsumadai, and their antioxidant, cytotoxicity, and antibacterial activities", RSC Adv., 7, pp. 39842-39851 (2017).

30. Jain, S. and Mehata, M.S. "Medicinal plant leaf extract and pure flavonoid mediated green synthesis of silver nanoparticles and their enhanced antibacterial property", Sci Rep., 7, p. 15867 (2017).

31. Ruiz-Baltazar, A.D.J., Reyes-Lopez, S.Y., Larranaga, D., et al. "Comparative study of Ag nanostructures: molecular simulations, electrochemical behavior, and antibacterial effect", J Nanomater., 2016, pp. 1-9 (2016). 
32. Devi, T.B., Ahmaruzzaman, M., and Begum, S. "A rapid, facile and green synthesis of $\mathrm{Ag} @ \mathrm{AgCl}$ nanoparticles for the effective reduction of 2, 4-dinitrophenyl hydrazine", Nouv J Chim., 40, pp. 1497-1506 (2016).

33. Niraimathi, K.L., Lavanya, R., Veerappan, S., et al. "Green synthesis and characterization of silver nanoparticles from aqueous extract of basella alba and their in-vitro antioxidant potentials", Int J. Pharm Sci., 6, pp. 393-396 (2014).

34. Khalil, M.M.H., Ismail, E.H., El-Baghdady, K.Z., et al. "Green synthesis of silver nanoparticles using olive leaf extract and its antibacterial activity", Arabian J. Chem., 7, pp. 1131-1139 (2014). DOI: https://doi.org/10.1016/j.arabjc.2013.04.007

35. Bayat, M., Zargar, M., Astarkhanova, T., et al. "Facile biogenic synthesis and characterization of seven metal-based nanoparticles conjugated with phytochemical bioactives using fragaria ananassa leaf extract", Molecules., 26(10), p. 3025 (2021).

36. Kanagamani, K., Muthukrishnan, P., Ilayaraja, M., et al. "Synthesis, characterisation and DFT studies of stigmasterol mediated silver nanoparticles and their anticancer activity", J. Inorg. Organomet. Polym., 28, pp. 702-710 (2018).

37. Khayati, G.R., Janghorban, K., and Shariat, M.H. "Isothermal kinetics of mechanochemically and thermally synthesized Ag from $\mathrm{Ag}_{2} \mathrm{O}$ ", Trans. Nonferrous Met. Soc., 22(4), pp. 935-942 (2012).

38. Clayden, J., Greeves, N., Warren, S., et al., Organic Chemistry, Oxford Univ. Press, Oxford, p. 585 (2001).

\section{Biographies}

Amineh Shafaei is currently a $\mathrm{PhD}$ candidate in Materials Science at Shahid Bahonar University of Kerman. She received her MSc and BSc degrees in Materials Science and Engineering in 2014 and 2010, respectively, from the Department of Materials Science and Engineering, Shahid Bahonar University of Kerman (Iran). Since 2014, she has been employed at Shahid Bahonar Company. She works as R \& D expert in the domain of copper and copper alloy semifinished fabricator and is currently an engineer in the shaping process

Gholam Reza Khayati is currently a faculty member at the Department of Materials Science and Engineering at Shahid Bahonar University of Kerman. He received his $\mathrm{PhD}$ in Materials Science from Shiraz University (Iran) and MSc (honor student) in Materials Science and Engineering at the Department of Materials Science \& Engineering, Tehran University (Iran). He received his BSc in Materials Science from Shahid Bahonar University of Kerman, Iran in 2004. He has published more than 40 scientific papers in the field of synthesis and characterization of nanostructures materials.

Reyhane Hoshyar is a faculty member at Cellular and Molecular Research Center, Department of Biochemistry, Faculty of Medicine, Birjand University of Medical Sciences, Birjand, Iran. She is currently doing her postdoc at Michigan State University of USA. 